\section{Implications of Arctic air pollution}

\author{
from S. A. Penkett
}

IN winter, the southward movement of the polar front allows air from polluted regions of the Northern Hemisphere to be transported into the Arctic, giving rise to a phenomenon known as 'Arctic haze'. This can lead to a visibility in this pristine area of the Earth's atmosphere of as low as $5 \mathrm{~km}$. The scientific community has been aware of the phenomenon for several decades but a detailed study has been impeded by the remoteness and inhospitable nature of the region. Recently, a series of 28 papers has been published (Geophys. Res. Lett. 5; 1984), describing experimental data obtained in a three-dimensional coverage of the atmosphere from Alaska to northern Norway. The papers deal with the meteorology that causes the phenomenon, and with many other aspects which have relevance to such topical concerns as the long-range transport of acidity and climatic change caused by anthropogenic activity.

The results mostly come from the Arctic Gas and Aerosol Sampling Program. It involved the use of six aircraft flying over the Arctic, collecting information on the nature and concentration of the particles making up the haze and on the composition of the associated air. The programme was organized by Russell C. Schnell from the University of Colorado. Scientists came from the United States and Europe, with the majority of the Europeans coming from the Norwegian Institute for Air Research. Funds were provided by the National Oceanic and Atmospheric Administration and by the British Petroleum Company. It is an example of the sort of cooperative programme that has to be mounted if air pollution problems of regional or global significance are to be tackled in detail.

Previous studies at ground level had shown that the aerosol consists mainly of fine particles in a relatively narrow size range $(0.2-0.4 \mu \mathrm{m})$. Its major constituent is sulphate, but because of the presence of substantial amounts of black carbon, it has been referred to as a 'combustion' aerosol. The major source areas are Europe and north-west Asia and the intensity is greatest in March and April. The aircraft surveys took place during this period and showed the presence of a complex layered structure, with well defined 'fronts' dividing clean and polluted air. The structural discontinuities are very similar to those found at the boundaries of cirrus clouds, suggesting high thermal stability and lack of turbulence throughout much of the Arctic atmosphere in winter conditions.
The origin of the fine particulate is thought to be anthropogenic but new evidence is presented of the possible volcanic origin of the giant $(>2 \mu \mathrm{m}$ diameter) particles. These are spread uniformly at latitudes from $0.3 \mathrm{~km}$ to $5 \mathrm{~km}$ and are not correlated with the haze layers.

Much of the sulphate in the haze layers is in the form of sulphuric acid and by contrast with other anthropogenic aerosols collected in urban areas of Europe and the United States, there is very little nitrate. The same is also true of the composition of snow samples collected in Spitzbergen.

The low abundance of nitrate is very intriguing. It could indicate that the aerosol is directly injected from combustion processes in the source regions and, indeed, evidence is presented to show that during the Arctic winter, particulate sulphate is not formed by atmospheric gasto-particle conversion on any significant scale. (That is perhaps not unexpected because of the almost complete absence of any atmospheric photochemistry in the region during the winter months.) A photochemical process may be possible, however, in the source regions at lower latitudes, in March and April. A thorough chemical study of Arctic air pollution may shed light on acid formation in the atmosphere in general. Specifically, measurements of nitrogen oxides and peroxyacetylnitrate, as well as sulphur dioxide, would define the emission characteristics of the source region and the nature of the chemical reactivity of its atmosphere in early spring and late winter.

The geographical origin of the air carrying the haze components has been investigated in several ways. One is by analysing the distribution of trace metals. High concentrations of elements such as nickel, lead and zinc may be traceable to smelters at particular locations deep inside the Soviet Union.

Another specific source of particulate material was identified in aircraft flights across a fold in the tropopause: volcanic debris was found to be entering the troposphere from the stratosphere in March 1983, presumably from the eruption of El Chichón in 1982. Considerable evidence of the influence of volcanoes on the particulate content of the Arctic atmosphere is stored in ice cores from Greenland. By contrast with the measurements reported for Spitzbergen, the Greenland ice core data suggest the presence of summer maxima in nitrate concentrations. Extensive studies of the composition of the ice in different parts of the Arctic could provide interesting details on the distribution of source areas.

Measurements of many gaseous species, including sulphur dioxide, ozone, halocarbons and hydrocarbons, are reported. Aircraft measurements show rather small fluctuations in the ozone vertical profiles, and no large changes within or outside the haze layers. The sulphur dioxide concentration peaks in phase with the aerosol in March-April. Large seasonal variations in the concentrations of many other gases were found. In winter, the Soviet Union seems to be a particularly abundant source of hydrocarbons, with the result that the concentrations of propane and butane are as high in March on Spitzbergen, as in air close to London in August. If these values are representative of the whole of the Arctic region, huge reservoirs of these gases could be built up during the winter to be consumed by tropospheric chemistry at lower altitudes as meteorological and sunlight conditions allow. This would probably lead to the production of large amounts of ozone in the troposphere of the Northern Hemisphere and may account for some of the interhemispheric asymmetry observed for this important trace gas.

The profusion of measurable trace gases, including five distinct brominecontaining species, and the absence of photochemical change in the Arctic winter, may allow a specific categorization of air mass sources. It is not unlikely that their relative concentrations will act as a 'fingerprint' for a source area as with no change in distribution during transport, the 'fingerprint' will remain intact over airmass tracks of many thousands of miles. A detailed examination could form the basis of an effective large-scale tracer experiment.

Many papers deal with the climatological consequences of the Arctic haze. The planetary albedo can be changed significantly by modification of light scattering, such that an increase of 2.5 per cent over the oceans and a decrease of 9 per cent over the icecap is predicted. The presence of graphite particles in the haze layers (at concentrations within a factor of 2 of those recorded in some cities in the United States) has a substantial effect on the optical depth of the atmosphere and is predicted to increase the energy absorbed by the Earth-atmosphere system by as much as 5 per cent. The climatic effects of aerosols of this type, injected into the atmosphere as a result of a large-scale nuclear exchange, are of much current interest. Fortunately, the Arctic haze phenomenon is on a much smaller scale, but studying it may provide quantitative data which can be used to test predictions of the temperature changes likely to be experienced in the aftermath of a large-scale nuclear war.

S.A. Penkett is in the Environmental and Medical Sciences Division, AERE Harwell, Oxfordshire OXI1 ORA. 